\title{
Esophageal cancer: Is the CROSS strategy ready for history books?
}

Matheus De Silva Sewastjanow, MD, ${ }^{\mathrm{a}}$ Jane E. Rogers, PharmD, ${ }^{\mathrm{b}}$ Wayne L. Hofstetter, MD, ${ }^{\mathrm{c}}$ and Jaffer A. Ajani, MD

\section{ABSTRACT}

The CROSS trial group deserves enormous credit for completing a well-powered randomized trial that has established the CROSS strategy as a standard of care for patients with potentially resectable esophageal cancer. However, the 10-year results are rather disappointing with only $38 \%$ of all patients treated with the CROSS strategy cured compared with approximately $25 \%$ who had surgery alone. Another standard, perioperative chemotherapy has produced similar disappointing results as the CROSS strategy. Although many of us are consumed by the question as to which option is better for our patients, we conclude that both strategies produce only marginal benefits. We should have better treatment options. The timing may be opportune to reflect on how to develop novel and rational strategies rather than propagate the historical empiric approaches. (J Thorac Cardiovasc Surg 2023;165:901-5)

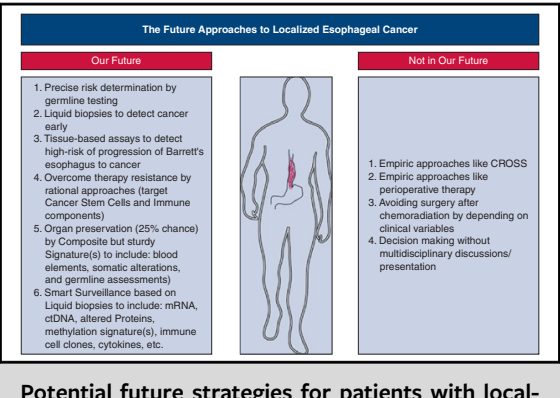

Potential future strategies for patients with localized EC.

\section{CENTRAL MESSAGE}

The CROSS trial group deserves enormous credit, but the timing may be opportune to reflect on how to develop novel and rational strategies rather than propagate the historical empiric approaches.

This Invited Expert Opinion provides a perspective on the following paper: J Clin Oncol. 2021 Apr 23;JCO2OO3614. https://doi.org/10.1200/JCO 20.03614. Online ahead of print.

See Commentary on page 906.
Feature Editor's Introduction-Optimal neoadjuvant treatment for esophageal and esophagogastric junction cancer remains elusive, but the CROSS trial greatly influenced the practice pattern of standard induction treatment of esophageal cancer since 2012 when results were published in The New England Journal of

From the Departments of ${ }^{\mathrm{a}}$ Gastrointestinal Medical Oncology, ${ }^{\mathrm{b}} \mathrm{Pharmacy}$, and ${ }^{\mathrm{c}}$ Thoracic and Cardiovascular Surgery, University of Texas M. D. Anderson Cancer Center, Houston, Tex.

Received for publication Oct 11, 2021; revisions received Nov 30, 2021; accepted for publication Dec 6, 2021; available ahead of print Jan 22, 2022.

Address for reprints: Jaffer A. Ajani, MD, Department of Gastrointestinal Medical Oncology, UT M. D. Anderson Cancer Center, 1515 Holcombe Blvd, Houston, TX 77030 (E-mail: jajani@mdanderson.org).

J Thorac Cardiovasc Surg 2023;165:901-5

$0022-5223 / \$ 36.00$

Copyright (c) 2022 by The American Association for Thoracic Surgery

https://doi.org/10.1016/j.jtcvs.2021.12.054
Medicine. This study randomized patients with resectable tumors to chemotherapy (5 weeks of carboplatin/ pacitaxel) concurrently with radiation $(41.4 \mathrm{~Gy})$ followed by surgery $(n=178$ vs surgery alone $n=188)$. OS was significantly better in the chemoradiation + surgery group (hazard ratio, 0.657; confidence interval, 0.495-0.87; $\mathrm{P}=.003)$. After wide adoption of the "CROSS strategy" spanning multiple continents, the CROSS study group summarized 10-year outcomes of the original trial in the Journal of Clinical Oncology in June of 2021. The results were sobering: The absolute cure rate was only $38 \%$ for the experimental group and $25 \%$ for the surgery alone group. Distant failure was the primary cause of death in both groups. Despite a complete pathologic response rate of $29 \%$ in the experimental arm (49\% for squamous cell histology), long-term survival was disappointing. The 
chemotherapy implemented in the CROSS trial was well tolerated $(91 \%$ completion rate preoperatively) but significantly underperformed in treating micrometastatic disease. Sewastjanow and colleagues were invited to comment on the 10-year summary of CROSS and place it into context in the most contemporary era of induction strategies for resectable esophageal or esophagogastric junction cancer. The landscape for neoadjuvant therapy has greatly evolved since CROSS and more potent induction chemotherapies (FLOT, FOLFOX) have been implemented before a "CROSS strategy." Surgery remains the cornerstone of optimal treatment for this disease, and standardization of surgical technique is paramount in clinical trials. Circulating tumor DNA analysis for detection of minimal residual disease after neoadjuvant therapy is a promising modality that may influence how surgery is used in the future. Last, the observed survival improvement with the adjuvant use of check point inhibition (CheckMate 577) in resected esophageal cancer has strengthened the exploration of immunotherapy in the neoadjuvant space of esophageal cancer and has opened up a new era aside from a CROSS strategy.

\section{Michael Lanuti, MD}

\section{THE ERA OF THE CROSS}

We congratulate the CROSS Study Group for reporting 10-year follow-up of the CROSS study. ${ }^{1}$ In the CROSS study, 366 favorably selected (resectable, cancer length $\leq 8 \mathrm{~cm}$, weight loss $\leq 10 \%$, and age $<75$ years) patients were randomly assigned to preoperative chemoradiation or surgery alone. The preoperative chemoradiation cohort had an improved overall survival (OS) compared with surgery alone. At this follow-up juncture, the absolute cure rate was $38 \%$ for the preoperative chemoradiation arm compared with approximately $25 \%$ for the surgery alone $\mathrm{arm}$. Although the strategy of chemoradiation followed by surgery has been under investigation for a long time with many prior trials having had multiple shortcomings, the CROSS Study Group should get the credit for completing a well-powered trial to establish the CROSS strategy as one of the therapeutic standards in the western world (www.NCCN.org). One clear advantage of using the CROSS strategy is that the total dose of radiation is 41.4 Gy in 23 fractions. The reduced number of fractions and shorter course of therapy improve compliance/tolerance without compromising efficacy.

\section{CROSS OR DON'T CROSS?}

The other standard approach for localized esophageal cancer (EC) is perioperative chemotherapy. More recently, the 5-fluorouracil, leucovorin, oxaliplatin, and docetaxel (FLOT) regimen has gained some popularity. ${ }^{2}$ In this study, chemotherapy with FLOT was compared with epirubicin and cisplatin (EC) plus a fluoropyrimidine. The study was confined to several German centers and 716 patients were randomized, an achievement. At the median follow-up of less than 3 years, the median OS (the primary end point) was 50 months for FLOT and 35 months for epirubicin, cisplatin, 5-fluorouracil/epirubicin, cisplatin, capecitabine (hazard ratio, 0.77; 95\% confidence interval, 0.63-0.94; $P=.012)$. FLOT resulted in considerably more grade 3 or 4 infections, neutropenia, diarrhea, and neuropathy than its control. Less than $50 \%$ of patients could complete the stipulated postoperative chemotherapy, and the pathologic responses were rather poor. The cure rate (projected at 5 years) appears to be less than $40 \%$ even though approximately $50 \%$ of patients had gastric primary cancer, and we know that patients with localized gastric cancer tend to have higher cure rates than patients who have cancer at the gastroesophageal junction or higher in the thoracic esophagus. ${ }^{3,4}$ Thus, the latest perioperative approach does not infuse a great deal of enthusiasm for adoption over CROSS but remains an option. Nevertheless, the current landscape of therapy of localized EC is based on practice preferences. Surgery remains central for achieving high cure rates. The question is being asked, which of the 2 strategies is superior for our patients?

Efforts are under way to answer this question. Currently, 2 major studies have been launched (ESOPEC NCT02509286, randomizing 438 patients, and NeoAEGIS NCT0172645, randomizing 377 patients), and the early data from Neo-AEGIS show that the 3-year estimated cure rates are similar for both strategies, approximately $56 \%{ }^{5}$ The NExT trial (JCOG1109) is comparing 3 arms: (1) preoperative cisplatin/fluorouracil, (2) preoperative cisplatin/fluorouracil/docetaxel, and (3) cisplatin/fluorouracil with radiation (41.4 Gy) as preoperative therapy. This Japanese study is appropriately focused only on squamous cell carcinoma of the esophagus (ESCC). All of these studies will produce useful information and hopefully answer the question.

At this time, it would suffice to state that the choice of preoperative chemoradiation or perioperative chemotherapy is a matter of one's preference (https://www.nccn. org/professionals/physician_gls/pdf/esophageal.pdf; accessed August 21, 2021). We find no compelling reason to favor one over the other, except that CROSS has a shorter treatment duration and intensive FLOT is associated with considerable toxicity and low therapy completion rate after surgery. An effective systemic approach might control eventual metastases better in many patients compared with chemoradiation.

One clear benefit of using the CROSS strategy is that we can implement the positive results of Checkmate 577. Checkmate 577 was a large global, multicenter trial that demonstrated the value of nivolumab in the adjuvant setting. ${ }^{6}$ Patients who had EC (ESCC or adenocarcinoma; 
esophageal adenocarcinoma [EAC]) and residual cancer in the resected specimen (in the primary and/or nodes) after trimodality therapy were randomly assigned to nivolumab for 1 year or placebo (2:1 randomization in favor of nivolumab). Disease-free survival (DFS) was the primary end point of the study, and OS was the secondary end point among others. 532 patients received nivolumab and 262 received placebo. At the median follow-up time of 24.4 months, DFS was significantly longer for nivolumab treated patients versus placebo-treated patients (hazard ratio, $0.69 ; 95 \%$ confidence interval, $0.56-0.86 ; P<.001$ ). The median DFS was 22.4 months for the nivolumab arm and 11 months for the placebo arm. The OS results are awaited. The US Food and Drug Administration has already approved nivolumab in the adjuvant setting. Therefore, if one would like to take advantage of the positive results of CheckMate 577, then the CROSS strategy could be preferred until we can have similar benefits from adjuvant immunotherapy in patients who receive perioperative chemotherapy.

\section{THERAPY RESISTANCE}

ECs (EAC or ESCC) are inherently resistant to therapy. ${ }^{7-9}$ This is evident in multiple trials reported using the preoperative chemoradiation strategy but more pronounced when using the perioperative chemotherapy strategy. ${ }^{1,2,10}$ When one administers preoperative chemoradiation, the pathologic complete response rate is approximately $25 \%,{ }^{10}$ meaning most patients have residual resistant cancer. However, with the use of perioperative chemotherapy, the pathologic complete response rate is only approximately $5 \%$. It is understood that a higher rate of pathologic complete response from to CROSS does not translate to an OS advantage compared with perioperative chemotherapy. Additionally, all novel strategies should be implemented and validated at the research centers before used widely.

Approximately $15 \%$ to $20 \%$ of EACs have signet ring cells (SRCs), and the mere presence of SRCs is associated with the poor prognosis (resistance to therapy) of patients with EAC. ${ }^{10-13}$ It is unclear at the moment what these SRCs represent. Differentiated cells from cancer stem cells (CSCs) or these cells implement unique resistance pathway(s) that cannot be overcome by the current standard approaches. In one study from our group focused on the molecular profile of patients with a better prognosis, the mere presence of SRCs trumped all other features (loss of chromosome 4 or higher APBEC enzyme signature). ${ }^{13}$ Efforts are needed to further characterize molecular underpinnings of SRCs to discover unique targets.

Additionally, particularly in EAC, cancer metabolism reflected by the degree of glucose uptake measured by positron emission tomography appears important. We focused on total lesion glycolysis (TLG) and maximum standardized uptake value. ${ }^{14}$ We studied 266 patients with EAC and discovered that the higher rate of metabolism (reflected by high TLG and maximum standardized uptake value) was associated with larger tumors, higher clinical stage, and higher TNM components. All of these patients received definitive chemoradiation, and only 60 patients were "cured." In the multivariate analysis, low TLG was independently associated with cure. Thus, imaging studies combined with clinical and molecular features may provide an opportunity to develop a signature that could help select appropriate therapy for a patient. Other positron emission tomography strategy (CALGB 80803) was recently published and will need further development. ${ }^{15}$

Many studies have documented resistance to therapy (chemotherapy and or chemoradiation) in the presence of CSCs and enrichment of CSCs in the residual resistant cancer. $^{13,16-20}$

Will we ever be able to customize approach for an individual patient? Our tools are limited, but the future appears bright (Figure 1). Although the technology has advanced, it is still not practical to receive comprehensive profiling results in a timely manner. The interpretation of such results can be daunting, but the limitations of various DNA platforms are substantial and evolving. Multiplatform profiling (including liquid biomarkers/tumor suppressor genes/immune components; Figure 1) is still not practical for therapy selection. How all this might shape up remains unclear, but we must strive to abandon the high level of empiricism that has permeated our clinical research strategies.

\section{ORGAN PRESERVATION}

One of the major challenges is how to preserve the esophagus of a patient with EC? Such an idea is fueled by 2 issues: (1) Surgery for EC is life-altering; and (2) there is a fraction of patients who have exquisitely sensitive cancer that is eradicated by preoperative therapy. Currently, the chemoradiation strategy appears more relevant for organ preservation than the perioperative chemotherapy strategy. The decision not to proceed with surgery is based on clinical intuitions or multidisciplinary expert decisions. A nomogram based on the clinical variables has been reported to predict pathologic complete response, meaning why operate on a patient to find no residual cancer in the surgical specimen, but such models have not been sturdy and not clinically implementable. ${ }^{21}$ Some biomarkers have been associated with ${ }^{22,23}$ chemoradiation resistance/response, but again, to develop a sturdy model, one would need more elaborate data and cooperative efforts. ${ }^{21}$

\section{LET US COLLECTIVELY KICK THE EMPIRICISM HABIT}

Most oncologists are trained to group patients in a given stage or stage range (eg, for EC, it could be from stages II to 
Our Future

1. Precise risk determination by germline testing

2. Liquid biopsies to detect cancer early

3. Tissue-based assays to detect high-risk of progression of Barrett's esophagus to cancer

4. Overcome therapy resistance by rational approaches (target Cancer Stem Cells and Immune components)

5. Organ preservation ( $25 \%$ chance) by Composite but sturdy Signature(s) to include: blood elements, somatic alterations, and germline assessments)

6. Smart Surveillance based on Liquid biopsies to include: mRNA, ctDNA, altered Proteins, methylation signature(s), immune cell clones, cytokines, etc.

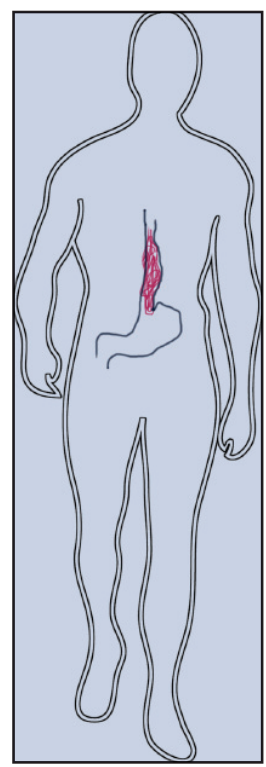

Not in Our Future

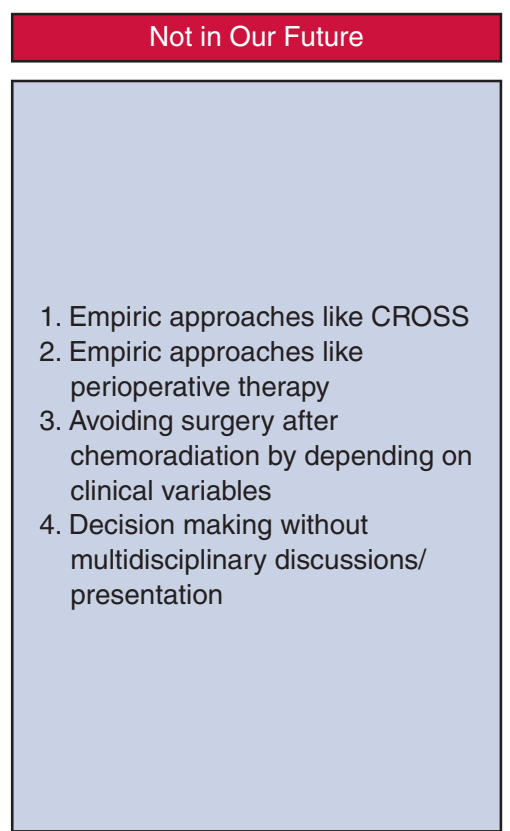

FIGURE 1. Potential future strategies for patients with localized EC.

III) and then treat all those patients similarly without regard to the tumor's genetic makeup or the patient's germline data. This old approach has not served us well. The entire empiric strategy triggered many large trials without adequate segmentation in patient population based on various elements. Individualizing therapy remains a difficult challenge because our infrastructures are not tuned into rapid testing. EAC with SRCs could be a model to begin with or highly metabolic EACs. In patients with SRCs, the results with the CROSS strategy or the perioperative strategy have been dismal, but we keep using these treatments. Is it due to the lack of idea/resources/motivation or a component of strategy fatigue? We are not sure which of these are leading our thinking. However, if we can produce better results in EAC with SRCs or hypermetabolic EAC, then advantages in other phenotypes could follow.

\section{CONCLUSIONS}

Progress against EC has been slow. We have arrived at a culture of performing (and demanding) excellent baseline staging and multidisciplinary evaluations. These are welcome advances. High-volume centers and highvolume surgeons have helped to reduce mortality/ morbidity. However, at the same time, we have become experts in empiricism. The time is now for all of us to take up a new challenge and find novel therapeutic strategies for patients with localized EC and produce better outcomes and let the contemporary strategies collect dust.

\section{Conflict of Interest Statement}

J.A.A. is an ad hoc paid advisor to Bristol-Myers Squibb company. MD Anderson Cancer Center receives funds from Bristol-Myers Squibb for clinical trials. All other authors reported no conflicts of interest.

The Journal policy requires editors and reviewers to disclose conflicts of interest and to decline handling or reviewing manuscripts for which they may have a conflict of interest. The editors and reviewers of this article have no conflicts of interest.

\section{References}

1. Eyck BM, van Lanschot JJB, Hulshof M, van der Wilk BJ, Shapiro J, van Hagen $\mathrm{P}$, et al. Ten-year outcome of neoadjuvant chemoradiotherapy plus surgery for esophageal cancer: the randomized controlled CROSS trial. J Clin Oncol. 2021;39:1995-2004.

2. Al-Batran SE, Homann N, Pauligk C, Goetze TO, Meiler J, Kasper S, et al. Perioperative chemotherapy with fluorouracil plus leucovorin, oxaliplatin, and docetaxel versus fluorouracil or capecitabine plus cisplatin and epirubicin for locally advanced, resectable gastric or gastro-oesophageal junction adenocarcinoma (FLOT4): a randomised, phase 2/3 trial. Lancet. 2019;393:1948-57.

3. Sano T, Coit DG, Kim HH, Roviello F, Kassab P, Wittekind C, et al. Proposal of a new stage grouping of gastric cancer for TNM classification: International Gastric Cancer Association staging project. Gastric Cancer. 2017;20:217-25.

4. Lopez A, Harada K, Chen HC, Bhutani MS, Weston B, Lee JH, et al. Taxanebased or platinum-based combination chemotherapy given concurrently with radiation followed by surgery resulting in high cure rates in esophageal cancer patients. Medicine (Baltimore). 2020;99:e19295.

5. Reynolds JV, Preston SR, O'Neill B, Lowery MA, Baeksgaard L, Crosby T, et al. Neo-AEGIS Study: Preliminary results of phase III RCT of CROSS vs. perioperative chemotherapy (MAGIC or FLOT). Proceedings of the American Society of Clinical Oncology. Virtual: ASCO; 2021. 
6. Kelly RJ, Ajani JA, Kuzdzal J, Zander T, Van Cutsem E, Piessen G, et al. Adjuvant nivolumab in resected esophageal or gastroesophageal junction cancer. $N$ Engl J Med. 2021;384:1191-203.

7. Shapiro J, ten Kate FJ, van Hagen P, Biermann K, Wijnhoven BP, van Lanschot JJ. Residual esophageal cancer after neoadjuvant chemoradiotherapy frequently involves the mucosa and submucosa. Ann Surg. 2013;258: 678-89.

8. Chirieac LR, Swisher SG, Ajani JA, Komaki RR, Correa AM, Morris JS, et al. Posttherapy pathologic stage predicts survival in patients with esophageal carcinoma receiving preoperative chemoradiation. Cancer. 2005;103:1347-55.

9. Cheedella NK, Suzuki A, Xiao L, Maru DM, Taketa T, Sudo K, et al. Association between clinical complete response and pathological complete response after preoperative chemoradiation in patients with gastroesophageal cancer: analysis in a large cohort. Ann Oncol. 2013;24:1262-6.

10. Blum Murphy M, Xiao L, Patel VR, Maru DM, Correa AM, Amlashi FG, et al. Pathological complete response in patients with esophageal cancer after the trimodality approach: the association with baseline variables and survival-The University of Texas MD Anderson Cancer Center experience. Cancer. 2017;123: 4106-13.

11. Corsini EM, Foo WC, Mitchell KG, Zhou N, Maru DM, Ajani JA, et al. Esophageal adenocarcinoma with any component of signet ring cells portends poor prognosis and response to neoadjuvant therapy. J Thorac Cardiovasc Surg. 2021;162:1404-12.e2.

12. Patel VR, Hofstetter WL, Correa AM, Agarwal A, Rashid A, Bhutani MS, et al. Signet ring cells in esophageal adenocarcinoma predict poor response to preoperative chemoradiation. Ann Thorac Surg. 2014;98:1064-71.

13. Hao D, He S, Harada K, Pool Pizzi M, Lu Y, Guan P, et al. Integrated genomic profiling and modelling for risk stratification in patients with advanced oesophagogastric adenocarcinoma. Gut. 2021;70:2055-65.

14. Harada K, Wu CC, Wang X, Mizrak Kaya D, Amlashi FG, Iwatsuki M, et al. Total lesion glycolysis assessment identifies a patient fraction with a high cure rate among esophageal adenocarcinoma patients treated with definitive chemoradiation. Ann Surg. 2020;272:311-8.
15. Goodman KA, Ou FS, Hall NC, Bekaii-Saab T, Fruth B, Twohy E, et al. Randomized phase II study of PET response-adapted combined modality therapy for esophageal cancer: mature results of the CALGB 80803 (alliance) trial. J Clin Oncol. 2021;39:2803-15.

16. Sims-Mourtada J, Izzo JG, Apisarnthanarax S, Wu TT, Malhotra U, Luthra R, et al. Hedgehog: an attribute to tumor regrowth after chemoradiotherapy and a target to improve radiation response. Clin Cancer Res. 2006;12:6565-72.

17. Song S, Chen Q, Li Y, Lei G, Scott A, Huo L, et al. Targeting cancer stem cells with a pan-BCL-2 inhibitor in preclinical and clinical settings in patients with gastroesophageal carcinoma. Gut. 2021;70:2238-48.

18. Izzo JG, Malhotra U, Wu TT, Ensor J, Luthra R, Lee JH, et al. Association of activated transcription factor nuclear factor kappab with chemoradiation resistance and poor outcome in esophageal carcinoma. J Clin Oncol. 2006;24:748-54.

19. Luthra R, Wu TT, Luthra MG, Izzo J, Lopez-Alvarez E, Zhang L, et al Gene expression profiling of localized esophageal carcinomas: association with pathologic response to preoperative chemoradiation. J Clin Oncol. 2006 24:259-67.

20. Izzo JG, Correa AM, Wu TT, Malhotra U, Chao CKS, Luthra R, et al. Pretherapy nuclear factor-kappaB status, chemoradiation resistance, and metastatic progression in esophageal carcinoma. Mol Cancer Ther. 2006;5:2844-50.

21. Ajani JA, Correa AM, Hofstetter WL, Rice DC, Blum MA, Suzuki A, et al. Clinical parameters model for predicting pathologic complete response following preoperative chemoradiation in patients with esophageal cancer. Ann Oncol. 2012;23:2638-42.

22. Ajani JA, Wang X, Song S, Suzuki A, Taketa T, Sudo K, et al. ALDH-1 expression levels predict response or resistance to preoperative chemoradiation in resectable esophageal cancer patients. Mol Oncol. 2014;8:142-9.

23. Wadhwa R, Wang X, Baladandayuthapani V, Liu B, Shiozaki H, Shimodaira Y, et al. Nuclear expression of Gli-1 is predictive of pathologic complete response to chemoradiation in trimodality treated oesophageal cancer patients. Br J Cancer. 2017;117:648-55.

Key Words: CROSS, esophageal cancer, localized, trial 